\title{
The Role of Arousal and "Gating" Systems in the Neurology of Impaired Consciousness
}

\author{
Nicholas D. Schiff and Fred Plum \\ Department of Neurology and Neuroscience, Weill Medical College of Cornell University, New York, New York, U.S.A.
}

\begin{abstract}
Summary: A brief taxonomy of neurologic disorders resulting in global impairments of consciousness is presented. Particular emphasis is placed on focal injuries of subcortical structures that may produce disorders that are otherwise associated to large bilateral cortical injuries. A distinction between subcortical arousal and "gating" systems is developed. Both clinical and experimental studies are reviewed in the context of these disorders and their possible underlying mechanisms. Key Words: Awareness-Persistent vegetative state-Intralaminar thalamic nuclei-Akinetic mutism-Reticular formation.
\end{abstract}

Understanding the disorders of human consciousness provides an empirical first step toward understanding mechanisms underlying human awareness. The neurologist's view of consciousness is derived from reliable observations of brain structure and function that lead to implicit hypotheses. These hypotheses represent implied models that may be examined in light of many recent advances in clinical and experimental neuroscience. Impaired consciousness of a variety of kinds may result from focal brain injuries that induce widespread functional changes. Differences in the clinical features of these human disabilities illustrate the interdependent relationships of brain mechanisms underlying arousal, gating, and expression of several specific neuropsychologic traits. In many cases, brain damage is too severe to permit meaningful recovery, as exemplified by the vegetative state. In others, transient or permanent behavioral abnormalities precipitated by focal lesions may reveal the contribution of specific brain systems to the overall conscious state. Global disorders of consciousness include stupor and coma, vegetative states, akinetic mut-

Address correspondence and reprint requests to Dr. Nicholas D. Schiff, Department of Neurology and Neuroscience, Weill Medical College of Cornell University, 1300 York Avenue, New York, NY 10021. ism, absence and partial complex seizures, hyperkinetic mutism, and others. These disturbances can be acute, and relatively brief, or may persist as protracted states. The authors explore the neurology of impaired consciousness, developing a brief taxonomy of global disorders of consciousness, and placing these neurologic diseases in the context of the underlying anatomy and physiology of arousal and "gating" systems. Definition of consciousness: A definition closely following that of James (1890) is offered for the psychological dimension of consciousness:

At its least, normal human consciousness consists of a serially time-ordered, organized, restricted and reflective awareness of self and the environment. Moreover, it is an experience of graded complexity and quantity.

Arousal, attention, intention, memory, awareness, and mood/emotion are primary neuropsychological compo-

This manuscript is adapted from the Electronic Seminar target article: Schiff ND, Plum F. The neurology of impaired consciousness: global disorders and implied models. Association for the Scientific Study of Consciousness. http://athena.english.vt.edu/cgi-bin/netforum/ nic/a/l. Several additional clinical observations of the role of these systems, particularly in neurologic disorders that exhibit fluctuations, are discussed therein. 
nents of consciousness. Although it is self-evident that these components are interdependent, neuroimaging studies have increasingly confirmed classical neurologic observations that particular brain systems develop to process or to generate specific functions (Fracowiak et al., 1998; Johnson, 1997).

We introduce a clinically formulated organization of global disorders of consciousness. Global disorders result from impairment of several neuropsychological components and result in diffuse, severe, or total loss of meaningful behaviors. The anatomic substrates of these disorders are reviewed with a special emphasis on the possible role of particular subcortical structures in their expression. We develop a distinction between arousal systems and gating systems. Arousal is identified with the different functional states that characterize forebrain activation on the basis of brainstem modulation of corticothalamic systems (Steriade and Llinas, 1988). Gating is formulated herein as a set of selective processes that may facilitate transient long-range interactions of largescale brain networks. We omit discussion of the closely related but more fundamental topic of binding, which relates to cellular mechanisms that generate featureselective processing within the forebrain at the microcircuit level (Gray, 1999; Llinas et al., 1998; Singer, 1999; Von der Marlsberg, 1999). It is not yet clear whether neurologic observations will lend particular insight into these mechanisms.

\section{A BRIEF TAXONOMY OF GLOBAL DISORDERS OF CONSCIOUSNESS AND A REVIEW OF ANATOMIC SUBSTRATES}

Global disorders of consciousness include stupor and coma, the vegetative state, akinetic mutism, absence and partial complex seizures, delirium, dementia, and a more recently defined state of hyperkinetic mutism (Table 1).

TABLE 1. Global disorders of consciousness

\begin{tabular}{lccccccc}
\hline \multicolumn{1}{c}{ State } & Coma* & PVS & ASZ & AKM & HKM & CPS & DEL \\
\hline Arousal & - & + & + & + & + & + & + \\
Attention & - & - & - & + & $+/-$ & $+/-$ & $+/-$ \\
Intention & - & - & - & - & + & $+/-$ & $+/-$ \\
Memory & - & - & - & - & - & - & $+/-$ \\
Awareness & - & - & - & $-/ ?$ & $-/ ?$ & $+/-$ & $+/-$ \\
\hline
\end{tabular}

* In addition to coma, generalized tonic-clonic seizures, postictal unconsciousness, concussion, and asystolic syncope may be included in this column. PVS, persistent vegetative state; ASZ, absence seizure; AKM, akinetic mutism; HKM, hyperkinetic mutism; CPS, complex partial seizures; DEL, delirium; -, absent; +, present (in crude form for attention $[\mathrm{AKM}]$ and intention $[\mathrm{HKM}]) ;+/-$, incompletely expressed; -/?, apparently absent.
These disorders are prototypes for other global disturbances of consciousness observed in complex brain injuries that often generate a mix of the clinical features described in the following paragraphs.

\section{Coma}

Coma reflects a totally unconscious and unarousable brain state that results from acute pharmacologic anesthesia or physical injury to the brain's arousal mechanisms (see Plum and Posner [1982] for a comprehensive review of causes). Functionally, coma is characterized by unarousable unresponsiveness to internal or external stimuli. Eyes are closed and even the most vigorous exogenous stimulation cannot evoke awakening. Comatose subjects express neither understandable words nor sounds, nor do they localize correctly specific noxious stimuli applied to any part of the body. Although several components of the arousal system are identified (see Arousal and Gating Systems), only relatively large rostral dorsomedial pontine, mesencephalic, and paramedian thalamic lesions or global damage to the cerebral hemispheres can produce sustained coma (Plum, 1991). In the comatose state, one finds no evidence of awareness of self or environment, nor are cyclical state changes observed.

Most pathologic coma derives from relatively few causes, and include, in order of incidence, (1) brain trauma; (2) cerebral vascular damage, including large cerebral and brainstem ischemic stroke, acute ruptured cerebral aneurysms, and large cerebral or subtentorial hemorrhage; (3) severe anoxemia or asphyxia resulting from cardiac asystole, drowning, absence of atmospheric oxygen, or abrupt, severe pulmonary dysfunction; (4) acute intracranial inflammatory disease; (5) intentionally or accidentally inhaled or ingested sedatives or street drugs; and 6) several systemic or cerebral metabolic disorders.

Table 1 indicates a concomitant loss of all neuropsychological components incurred with this injury. Stupor is an imprecise term applied to patients with marked impairment of arousal who nevertheless can be sufficiently stimulated from their sleeplike condition to express purposeful, but often inconsistent, responses to their environment. In addition to coma, other forms of brief unconsciousness such as syncope, concussion, and brief generalized tonic-clonic seizures or postictal unconsciousness may be included in the left column of Table 1.

Syncope describes unconsciousness caused by severe, acute reductions of cerebral blood flow, and may be brief and benign or sustained and dangerous. Severe arterial 
hypotension, bringing critical capillary perfusion to less than $30 \mathrm{~mm} \mathrm{Hg}$ for more than 30 minutes or more, risks producing serious, potentially long-lasting brain injury. Permanent anoxic brain damage may begin as little as 2 to 4 minutes after the continuation of complete asystole. Concussion vaguely describes an acute traumatic brain injury that induces sudden unconsciousness but does not necessarily leave traces of either the specific type of trauma, the length of subsequent unconsciousness, or the immediately expected anatomic neuropathology of the brain. Acceptable quantitative information regarding underlying mechanisms is unavailable.

\section{Persistent Vegetative State (PVS)}

Among the most important contributions to our understanding of mechanisms generating arousal and sustained wakefulness in the past half century have been the discovery of the physiology of arousal by Moruzzi and Magoun (1949) and others (as described later), and the clinical identification by Jennett and Plum (1972) of the automatic and isolated sleep-wake function of the PVS. The vegetative state presents the fundamental clinical dissociation of arousal from all other components of consciousness (see Table 1). In the vegetative state, irregular cyclic arousal (absent in the comatose patient) recovers without evidence of awareness of self or the environment. The structural anatomic damage that can precipitate PVS varies widely (Adams et al., 1999; Multisociety Task Force on PVS, 1994). Autopsy examinations in large series of post-traumatic PVS subjects (Adams et al., 1999; Danze et al., 1989) show varying degrees of destruction/degeneration that affect bilaterally the cerebral cortex, the cerebral white matter, and sometimes the mesencephalic tegmental structures either independently or all together. The mesencephalic lesions mostly reflect damage secondary to early compression of the brainstem after swelling that results from brain injury (herniation). Postmortem studies of nontraumatically induced PVS have been fewer but also disclose multifocal bilateral cerebral lesions with or without severe destruction of basal ganglia or thalamus (Dougherty et al., 1981). In addition to cases of widespread damage resulting from anoxic or traumatic brain injury, vegetative states may also result from focal injuries to the paramedian brainstem and thalamus (Castaigne et al., 1981; Facon et al., 1958; Kinney et al., 1994; Plum and Posner, 1966; Relkin et al., 1990).

The clinical judgment of unconsciousness in PVS has been supported by positron emission tomographic (PET) studies that reveal overall cerebral metabolism to be reduced by $50 \%$ or more below the normal rate
(DeVolder et al., 1990; Levy et al., 1987; Rudolf et al., 1999; Tomassino et al., 1995). The observed levels are equivalent to those found in persons undergoing deep surgical anesthesia (Blacklock et al., 1987). Recently, along with our colleagues Drs. Urs Ribary and Rodolfo Llinas, we have documented behavioral and physiologic variations in a few patients in the vegetative state (Plum et al., 1998; Ribary et al., 1998; Schiff et al., 1999c). One of these patients randomly expressed occasional single, understandable words (Schiff et al. 1999c). The results of these patient's PET studies identified isolated islands of left cerebral structures that operated at an abnormally low metabolic rate but at nearly twice the rate of remaining brain. Similar isolated expressions have been encountered in several other vegetative patients. Typically, they express easily identifiable, stereotypic, emotionallimbic responses. These emotional expressions likely reflect distinct and isolated limbic mechanisms, possibly linked to integrative brainstem structures outside the thalamocortical systems that typically undergo overwhelming injury in patients in a PVS.

\section{Akinetic Mutism}

The term akinetic mutism covers different behavioral expressions that relate to damage of several cerebral and subcortical structures. There are two primary forms of akinetic mutism:

1. The hypervigilant form, which typically results from bilateral cortical injury of the anterior cingulate, the medial frontal cortex, or the orbitobasal cortex

2. The slow syndrome or mixed features form, which is associated to injury to the bilateral paramedian mesodiencephalic injury (mesencephalic reticular formation $[\mathrm{MRF}] /$ intralaminar nuclei $[\mathrm{ILN}] /$ periaqueductal gray matter), the bilateral globus pallidus, the bilateral caudate nucleus, or the medial forebrain bundle

Although sometimes confused with the vegetative state, akinetic mutism may resemble a state of constant hypervigilance. Such patients appear attentive and vigilant but remain motionless. The preservation of visual tracking in the form of smooth pursuit movements (or optokinetic responses) differentiates this condition from the vegetative state. Thus, classic akinetic mutism as listed in Table 1 reflects the recovery of a crude wakeful attentiveness without the apparent recovery of any other neuropsychological function.

The original term akinetic mutism was coined by Cairns et al. (1941) to describe a young woman who, 
although appearing wakeful, became mute, rigidly motionless, not spastic, and apparently unconscious, when a craniopharyngiomatous cyst expanded to compress the walls of her third ventricle and the posterior medialventral surface of the frontal lobe. When the cyst was drained, she recovered full awareness of the immediate present but had no memory of the previous events. Eye movements were not described in this young woman, but almost all recent cases of this type are said to display rare, slow, but seemingly attentive, conjugate eye movements. Oculocephalic stimulation in these patients may evoke limited fractions of lateral gaze. Subsequent observers have somewhat widened the anatomic sites that may generate the syndrome, based on similar behavioral patterns. These include selective or associated injury to the medial-basal prefrontal area including Cairn's zone, the medial forebrain bundle, the anterior cingulum, the medial prefrontal region supplied by the anterior cerebral arteries, and the pallidum and caudate nuclei. A similar picture, but not including absence of eye movements, can rarely be a feature of untreated, rigid Parkinson's disease. Recently, a few investigators have reported finding a strong clinical resemblance to this syndrome in the terminal state of prion disease (Otto et al., 1998). The hyperattentive form of this disorder is typically seen in patients with bilateral lesions of the anterior cingulate and mesial frontal cortices. Frequently, the state reflects frontal damage caused by rupture of an anterior communicating artery aneurysm (Nemeth et al., 1988). The associated injury may sometimes be accompanied by injury to the hypothalamus and anterior pallidum.

Subsequent to Cairn's original description, Castaigne et al. (1966) emphasized that patients with structural injuries affecting the medial-dorsal thalamus extending into the mesencephalic tegmentum experienced severe memory loss and apathetic behavior. Segarra (1970) described seven more examples of combined damage to the medial-caudal thalamus and medial-dorsal mesencephalon causing the same signs and symptoms as "akinetic mutism." Because of this unfortunate confusion of both behavior and structural lesions, these two related syndromes are often misidentified, and we prefer to distinguish the two types (as listed previously). A persistent dementia characterizes the recovery phase of this later disorder, which is often described as slow syndrome (Katz et al., 1987). Patients with slow syndrome appear apathetic and hypersomnolent but may speak with understandable words (Fisher, 1983). Most of these patients, however, are able to move and speak after they regain awareness. They are not semirigid, as are akinetic mute patients, and they lack the appearance of vigilance. Subcortical lesions that may produce slow syndrome include bilateral lesions of the paramedian anterior or posterior thalamus and basal forebrain (Castaigne et al., 1981; Facon et al., 1958), the MRF including periaqueductal gray matter (Segarra, 1970), caudate nuclei (or left caudate in isolation [Bhatia and Marsden, 1994]), globus pallidus interna (Helgason et al., 1988; Mega and Cohenour, 1997), or selective interruption of the medial forebrain bundle (Ross and Stewart, 1981).

The common denominator of all akinetic mute states can be related to the disabling of several parallel, segregated corticostriatopallidal-thalamocortical (CSPTC) loops that involve the frontal lobes either directly or indirectly (Mega and Cohenour, 1997). The most devastating injury is a bilateral loss of basal-mesial cortical tissue, after which little further recovery develops. Bilateral lesions of the globus pallidus interna are unusual in that this structure contains each of the identified CSPTC circuits involving the frontal lobe, striatum, globus pallidus, substantia nigra, and thalamus (Mega and Cohenour, 1997). Thus a bilateral pallidal injury can disable several parallel networks. At least partial cognitive function can recover after some bilateral injuries to the paramedian thalamus and mesencephalon (Castaigne et al., 1981; Segarra, 1970; Stuss et al., 1988; van Domburg, et al., 1996; and see the discussion in Schiff and Plum [1999]). Akinetic mutism resulting from injury to these structures is likely to reflect their unique role along with the nucleus reticularis of the thalamus in gating both these parallel CSPTC loops (Groenewegen and Berendse, 1994a, 1994b) and specific long-range corticalcortical interactions (as described later). Isolated injury to the periaqueductal gray region has also been described in experimental models of akinetic mutism (Panksepp, 1998; Watt, 1998). Selective injury to the medial forebrain bundle removes a strong dopaminergic modulation of medial frontal lobe structures, functionally downregulating these regions (Ross and Stewart, 1981). This loss of modulation is reversible, and can be corrected by giving patients dopaminergic agonists (Fleet et al., 1987).

\section{Hyperkinetic Mutism}

Hyperkinetic mutism describes a wakeful, continuous movement disorder accompanied by at least partial loss of self-awareness. It is a recently described disorder seen primarily with bilateral destruction of temporal parietal occipital junctions and wider lesions encompassing occipital-parietal regions (Fisher, 1983; Inbody and Jankovic, 1986; Mori and Yamadori, 1989). Inbody and Jankovic, in 1986, first applied the term hyperkinetic mutism to an elderly woman who first developed a unilateral facial dystonia and hemiballistic activity in her 
ipsilateral extremities. Three weeks later she collapsed and developed similar movements on the contralateral side, after which she became permanently "confused," and developed cerebral blindness and mutism. Noxious stimuli evoked only moans and no evidence of conscious intention. Bilateral ballistic movements continued until her death 4 months later. MRI identified "bilateral watershed" infarction of the occipital poles and both parietal areas, left greater than right.

Fisher (1983) described somewhat similar unconscious behavior in an elderly man with auricular fibrillation who abruptly developed "constantly threshing, churning ... (behavior, and being) ... apparently without mind." Autopsy disclosed bilateral parietal, temporal, and occipital infarction secondary to emboli lodging in the posterior sylvian branch of the middle cerebral arteries. Somewhat similar clinical behavior and autopsy or computed tomographic findings were described in four patients by Mori and Yamadori (1989). Similar expressions of unconscious, hyperactive behavior have also been described in patients with acute posterior cerebral leukoencephalopathies resulting from acute hypertension, autoimmune disorders, and inflammatory reactions to chemotherapeutic agents.

Thus, patients with hyperkinetic mutism exhibit totally unrestrained but coordinated motor activity in the absence of any external evidence of awareness of self or the environment. Their behavior strongly overlaps with the syndrome documented by Kluver and Bucy (1939)psychic blindness, hypermetamorphosis, hyperorality, emotional dyscontrol, and severe amnesia-described first in nonhuman primates after large, bilateral resections of the temporal lobe. Affected humans with similar damage demonstrate an inability to develop conditioned responses, and produce no apparent memory of self (Inbody and Jankovic, 1986). We identified one young woman with this condition following encephalitis who failed to recognize herself in a mirror (Gallup, 1983), and demonstrated constant, briefly directed exploratory reaching and grasping of objects without demonstrable understanding of their use (unpublished case). Of note, a similar state of mutism, hypermetamorphosis, and other features of the Kluver-Bucy syndrome have been described after selective, bilateral injury to anterior paramedian thalamic structures-most likely the central lateral and paracentral nuclei (see Muller et al. [1999], and the discussion in Schiff and Plum [1999]).

Hyperkinetic mutism is an opposite condition to akinetic mutism, with preserved unconscious expression of frontal intentional mechanisms; loss of sustained, directed attention presumably requiring posterior attentional components of the inferior parietal lobe or poste- rior temporal lobe; and a state of behavioral unawareness despite a whirlwind of activity. Taken together with the akinetic mute state, these patients demonstrate a global double dissociation of minimally expressed intention and attention. The two conditions hint that large, distributed cerebral networks underlying these neuropsychological components can be grossly dissociated-with varying degrees of impairment of the CSPTC loops leading to varieties of akinetic mutism and, conversely, the hyperkinetic mute state reflecting the relatively isolated activity of preserved CSPTC loops. Intention carries a special meaning, implying a particular, continuous quality of transitive, objective purpose that characterizes the awake human mind (Dennett, 1987; Haugeland, 1981; Searle, 1983). The fragment of intention expressed in the meaningless motor activity of the hyperkinetic mute patient is a reciprocal of the crude form of attention seen in akinetic mutism, and both examples reveal the fundamentally unconscious nature of such fragmentary neuronal activity. Similar examples include the repetitive, uncontrollable production of words in Tourette's syndrome (similar to the vegetative patient described earlier) and other movement disorders. We have identified a hyperkinetic vegetative patient who expresses continuous, nontargeted but coordinated movements (Plum et al., 1998). Cerebral metabolic studies in this patient reveal the isolated expression of the parallel CPSTC loops damaged during the akinetic mute state (manuscript in preparation).

\section{Absence Seizures and Complex Partial Seizures}

Absence seizures and complex partial seizures both reflect unique alterations of consciousness, including global loss of consciousness during many absence seizures. These disorders share the features of attentional and intentional failure, loss of working memory, and intraictal perceptual dissociation. In their classic form, absence seizures represent momentary vegetative states (see Table 1). Long-lasting absence seizures and complex partial seizures demonstrate automatic behaviors that bear some resemblance to hyperkinetic mutism. Although debate surrounds the underlying mechanism of absence seizures, thalamocortical generation is indicated by both clinical and experimental studies of absence seizures (McCormick and Bal, 1997; Snead, 1995; Steriade and Contreras, 1995). The key role of corticothalamic projections in organizing large-scale, coherent EEG patterns has been well demonstrated in recent studies (Contreras et al., 1997). Most current animal models include the nucleus reticularis of the thalamus (NRT) in conjunction with thalamocortical relay cells 
and the cortex as the essential substrate for the cortical initiation of the seizure (Hugenard, 1999; Steriade and Contreras, 1995). In addition, the passage from the thalamus to the cortex must rely on specific and nonspecific relay nuclei that project to the cortex (the NRT does not project to the cortex, see Schiebel and Schiebel [1966]). Among these thalamic nuclei, the ILN play an important role in the genesis of these seizures. Experimental studies in guinea pig, cat, monkey, and human all demonstrate that generalized 3/s spike-waves, and an associated behavioral absence may be elicited by electrical stimulation of the intralaminar nuclei (David et al., 1982; Hunter and Jasper, 1949; Ingvar, 1955; Jasper and Drooglever-Fortuyn, 1947; Pollen et al., 1963; Steriade, 1974; Velasco et al., 1993). These studies show a robust reproducibility of the 3/s spike-and-wave phenomenon across species and behavioral state. In addition, an important role for brainstem reticular contributions to this seizure has also been argued (Gloor and Fariello, 1988; Jasper, 1991).

Taken together, an overlap exists between the anatomic structures involved in absence seizures and those that, when injured, induce coma, vegetative states, and akinetic mutism. Absence seizures represent one of a few conditions that may produce brief unconsciousness without any evidence of lasting structural injury. Unlike concussions, syncope, or pharmacologic anesthesia, arousal is preserved during absence seizure, demonstrating the selective loss of integrative functions with these events (see the discussion later in this article of early centrencephalic theories).

Complex partial seizures of the temporal lobe vary in the quality and degree of their alteration of consciousness. Their clinical features, however, are sometimes indistinguishable from absence seizures (Delgado-Escueta et al., 1982). Based on distinct pharmacologic responses, electroencephalographic patterns, and focal anatomic causes, absence and complex seizures are usually considered to have completely separate mechanisms (Mikati and Holmes, 1993). Nevertheless, some evidence exists that overlapping thalamocortical circuits may be involved in both seizure types (Fromm, 1986; Mraovitch and Calando, 1995, 1999; Schiff et al., 1999a). Specifically, the anterior thalamic ILN (paracentralis and possibly central lateral nuclei) are hypothesized to provide a thalamocortical contribution to this type of seizure activity (Schiff et al., 1999).

The loss of consciousness in absence seizures is now argued to result from the inhibition of more than half of the thalamocortical neurons, as a result of $\gamma$-aminobutyric acid-ergic NRT neurons following paroxysmal potentials arising in neocortical neurons (Steriade, 1999; Steriade and Contreras, 1995). Thalamocortical neurons take a passive role in absence seizures, inhibited indirectly by the neocortex (Steriade, 1999). This explanation, however, may leave out a selective aspect of the seizure discharge. Although very rare, there are welldocumented cases of patients with continuous absence seizures without intellectual impairment (Gokyigit and Caliskan, 1995). The EEG amplitudes during these seizures are comparable with those seen in patients with clinical epilepsy, and they demonstrate that the alteration in consciousness is not merely the result of a threshold level of cortical recruitment into the ongoing seizure. We speculate that the absence seizure reflects the transient hypersynchronization of selective cortical and subcortical systems that gate integration of segregated forebrain networks. This is suggested by the lack of a postictal depression in the classic form of the seizure, and the lower amplitude of the seizure discharges (compared with other forms of generalized seizures) that both hint that the disturbance may be confined to a selective system.

\section{Global Disorders of Consciousness with Partial Preservation}

\section{Delirium}

Delirium is generally perceived as primarily an attentional and working memory disturbance (Mesulam, 1983). A salient component of this functional disturbance, however, is temporal disorientation. A wide variety of disorders including acute illness, underlying dementia, and substance withdrawal can precipitate delirium (Plum and Posner, 1982). Few gross structural lesions reliably precipitate acute, agitated delirium and typically are restricted acute parietal lobe infarcts of the nondominant hemisphere or more rarely temporal lobe infarcts. An older less well-known set of observations points to the selective disturbance of autobiographic chronometry seen in some subtle cases of delirium. Several early reports of after-effects of bilateral medial thalamotomy indicate a similar state with a disturbance labeled chronotaraxis (Spiegel et al., 1956). We observed a similar phenomenon in a patient in whom it represented an early sign of bilateral paramedian thalamic and unilateral mesencephalic stroke (unpublished observations).

\section{Dementia}

Dementia is primarily a disturbance of, at first, shortterm, and later, long-term memory resulting from diffuse cortical dysfunction that evolves to affect each of the 
listed neuropsychological components. At the end stage, advanced dementia may be indistinguishable from the vegetative state. A detailed review of etiologies is beyond the scope of this paper. Several forms of subcortical dementia are also known and associated with injury to different structures including the basal ganglia, substantia nigra, ventral tegmental area, globus pallidus, and brainstem (Brown and Marsden, 1988; Cummings, 1986). Also included prominently among subcortical causes of dementia are infarctions of the thalamic ILN and degenerative illness leading to destruction of these and other thalamic nuclei (thalamic softening syndromes; see Martin, 1997, for a review).

\section{AROUSAL AND GATING SYSTEMS}

In all cases of selective lesions producing global disorders, unconsciousness appears to arise from either large bilateral injuries to frontal or posterior association cortices, or selective subcortical injuries. As noted earlier, the pattern of subcortical injuries suggests that the paramedian mesodiencephalic structures of the "classic" arousal system (the ILN of the thalamus and the MRF) may play a primary role in the loss of integration observed in these disorders. The following section discusses the current view of arousal systems and their possible distinction from structures that may be more appropriately considered "gating" systems.

\section{Arousal Systems}

The concept of brainstem arousal systems was introduced by the pioneering work of Morison and Dempsey (1942), Jasper and Droogleever-Fortuyn (1947), and Morruzzi and Magoun (1949). Initially, the role of the MRF and the thalamic ILN was emphasized as mediating arousal and setting the stage for sensory processing in higher integrative brain functions (Hunter and Jasper, 1949; Morruzi and Magoun, 1949; see the recent review by Jasper [1998]). Electrical stimulation of these mesodiencephalic structures demonstrated their role in both EEG desynchronization and behavioral arousal.

The classic interpretations of the arousal system have been incorporated into the current conception that identifies arousal as interdependent on the output of cholinergic, serotoninergic, noradrenergic, dopaminergic, and histaminergic nuclei located predominantly in the brainstem, basal forebrain, and posterior hypothalamus (Marrocco et al., 1994; McCormick, 1992; Steriade et al., 1997). Arousal is now viewed in terms of global modulations of the thalamocortical system that define specific functional states (McCormick, 1992; Steriade and Llinas, 1988).
Several studies have sought to determine how necessary or sufficient the individual the individual brainstem nuclei are, including the serotoninergic (median raphe) and noradrenergic (locus ceruleus) groups, without providing compelling evidence that any single group is indispensable (Dringenberg and Vanderwolf, 1997; Marracco et al., 1994; Steraide et al., 1997). Even within global modulatory states, increasing evidence identifies the fine structure contributed by selective activation of interdependent arousal systems (Coull et al., 1997; Marracco et al., 1994; Marracco and Davidson, 1998; Silberstein, 1995). For example, varying effects of noradrenergic, dopaminergic, and cholinergic neuromodulators have been identified in visuospatial attention paradigms (Marrocco and Davidson, 1998). The nucleus basalis has also been partitioned into regions selectively active in different behaviors (Sarter and Bruno, 2000). A substrate for complex interactions among these brainstem nuclei is provided by the increasingly high degrees of interconnection identified in anatomic studies of these neuronal populations (Smiley et al., 1999).

Cholinergic pathways that originate in the laterodorsal tegmental and pedunculopontine nuclei project rostrally to various targets and play a prominent role in most discussions of arousal mechanisms (see Steriade et al. [1997]). Their output is likely insufficient to generate normal arousal, which must require additional glutamatergic and other brainstem populations (Steriade, 1999), although a role in sharpening attention and modulation of conscious activity is well supported (Marracco and Davidson, 1998; Posner and Rothbart, 1998). Well-documented clinical reports indicate that selective damage to these pontine cholinergic nuclei can prevent or greatly reduce rapid eye movement and normal sleep, resulting in chronic hyposomnia, but not necessarily even a transient coma (Autret et al., 1988; see also Plum [1991] for further discussion and related findings in patients with olivopontocerebellar atrophy). Cognitive ability reportedly remains intact in persons not receiving more rostral brainstem damage (Lavie et al., 1984). Cholinergic nuclei located more rostrally in the basal forebrain influence cortical cognitive and memory functions as well as EEG desynchronization, but they seem to make no firmly established contribution to arousal per se. The selective contributions of dopaminergic (ventral tegmental area) and histaminergic (tubomammilary nucleus) agents have also been studied (Marracco et al., 1994).

\section{Centrencephalic Integration and Early Models of Forebrain Gating Systems}

In the early 1950s, Penfield and Jasper (1952, 1954) proposed a clinically engendered hypothesis involving 
brainstem arousal mechanisms and termed the process centrencephalic integration (see Jasper [1991, 1998] for historical review). Penfield drew his speculation from observing the temporary loss of consciousness accompanying absence seizures and from linking this unique paroxysmal event to a "highest level" brain mechanism that underlay conscious awareness. Most important, his hypothesis related the observed loss of consciousness to a specific failure of a generalized integrating mechanism. The model, never detailed precisely in anatomic terms, strongly emphasized the role of mesodiencephalic structures-specifically, the thalamic ILN and the adjacent MRF. The theory was criticized strongly, but nonscientifically, by conflating the hypothetical system with a locus (a "centrencephalon") in which such an integration underlying consciousness would be achieved (Penfield, 1958; Walshe, 1957; see also Thompson [1993]). A close reading of Penfield's (1958) writings, however, clearly indicates his concept that these mesodiencephalic structures organized a process that enabled a "synchronous central and cortical activity, activity in the brainstem and in those areas of the cortex of either hemisphere whose function is suited to the changing requirements of the moment" (p. 4) (Jasper, 1991).

Working with both alert and anesthetized cats, Skinner and Yingling (1977) pioneered a series of experiments that examined the integrative physiology of the MRF, the NRT, and the medial thalamic-mesial frontal cortical systems (including the ILN and related nonspecific thalamic nuclei). These investigators proposed that gating of attention was achieved by medial thalamofrontal cortical and MRF control of NRT inhibition of specific thalamic relay nuclei (Fig. 1B). The model proposed that intentionally directed action emanated from the frontal cortical-thalamocortical projections, with reflex orienting responses able to interrupt via the MRF pathway to the NRT. Schiebel (1980) enlarged on this model with a more anatomically detailed treatment of the MRF, and Crick (1984) further proposed that the reticular nucleus constructed the conscious "searchlight" of attention. Crick's proposal (1984) emphasized a role for the lowthreshold spike burst that results from NRT-mediated hyperpolarization of thalamocortical relay neurons. The inactivation of this conductance, however, during the depolarized states associated with wakefulness and attentive behaviors rules out this mechanism (see Steriade [1999]). Recent anatomic studies have detailed specific sectors within the NRT that could partition thalamocortical activation along the lines envisioned by these investigators (Guillery et al., 1998). Moreover, physiologic studies in rats demonstrate such a selective NRT role in attentional processing using a Posner task (Posner and Rothbart, 1998; Weese et al., 1999).

Recent human PET studies demonstrate MRF and ILN coactivation during attentional processing, and provide further support for the gating concept of selective attention (Kinomura et al., 1996). Kinomura et al. (1996) used PET scans to demonstrate increased regional blood flow in the MRF, ILN, and prefrontal, frontal, parietal, and primary sensory cortices when quiet wakefulness was compared with simple reaction-time tasks in a visual or somatosensory attention paradigm. A specific role for the thalamus in mediating interactions of attention and arousal has also been demonstrated recently in careful, state-controlled studies using functional MRI (Portas et al., 1998).

\section{ILN and Forebrain Gating}

Although the arousal systems per se are no longer considered to include the ILN, most of these brainstem nuclei, particularly the cholinergic and noradrenergic groups, innervate the ILN directly. Initially the ILN was modeled as a "nonspecific" system of ascending projections to the cortex that received ascending projections from the reticular activating system and provided widespread cortical activation (Moruzzi and Magoun, 1949). More recent anatomic studies have demonstrated that individual ILN subdivisions project specifically to selected cortical and subcortical regions (Berendse and Groenewegen, 1991; Bogen, 1997; Groenewegen and Berendse, 1994a, 1994b; Jones, 1998; Macchi and Bentivoglio, 1985, 1999). Macchi (1993) suggested a "dual" mode for the ILN that includes both transferring and modulating thalamocortical interactions. The close connections between the arousal systems and the ILN provide an anatomic substrate for the ILN to modulate different behavioral states within the state of wakefulness (Marrocco et al., 1994; Steriade et al., 1997).

The ILN incorporates a number of different cell groups located in the paramedian thalamus that have similar, widely dispersed, but relatively specific, projections to the cortex, as well as strong efferent projections to the basal ganglia (Groenewegen and Berendse, 1994b). Increasing evidence points to the selectivity of subdivisions of the ILN and related midline nuclei (Groenewegen and Berendse, 1994b; Macchi and Bentivoglio, 1999). The anterior group nuclei (including CL and $\mathrm{Pc}$ ) tend to project to posterior primary sensory and association cortices along with projections to prefrontal and posterior parietal and temporal regions. The central lateral nucleus projects to frontal eye fields, the dorsal lateral prefrontal cortex, and the anterior parietal associ- 
ation cortex (Leichnetz and Goldberg, 1988; Macchi and Bentivoglio, 1985). The paracentralis nucleus has strong connections with the temporal association cortex (Yeterian and Pandya, 1989). The posterior ILN group is dominated by the $\mathrm{Cm}-\mathrm{Pf}$ complex and projects strongly to the basal ganglia, premotor cortices, and the parietal cortex (Berendse and Groenewegen, 1991, 1994a, 1994b). The parafasicularis nucleus also projects to the frontal eye field and posterior parietal association cortex. Thus, the cortical and subcortical innervations of the ILN place them in a central position to influence distributed networks underlying arousal, attention, intention, working memory, and sensorimotor integration, including gaze control. One may further speculate that the anterior ILN groups perhaps have a greater role in sensory information gating within overall sensorimotor integration. Conversely, the posterior ILN group likely plays a key role related more to motor gating (see Mennemeier et al. [1997] and Groenewegen and Berendse [1994a]). Regional specificity for the ILN and related midline nuclei is being detailed further within specific corticothalamic networks (Van der Weerf and Mitter, 1999). A related finding observed in a multidimensional scaling model of the entire cat thalamocortical system (Scannell et al., 1999 ) is that the ILN (CL, Pc, Cm-Pf) have the unique property of clustering, centered among all other segregated thalamocortical loop systems (frontal, limbic, temporal, and parietal).

Each cytoarchitecturally defined cell group within the ILN has a broad range of cortical targets, but this is not the case for individual neurons. Unlike neurons of the specific thalamic relay nuclei, neurons of the ILN synapse in layer I on the apical dendrites of pyramidal cells in layers II through III, and layers V and VI (Macchi, 1993). Following this input pattern, ILN stimulation activates both the supragranular and infragranular layers (Sukov and Barth, 1998). Jones (1998a, 1998b) has recently redefined thalamic subdivisions on the basis of subcellular characteristics of the neurons: Those with widespread superficial projections are called matrix components and are composed of calbindin cells, and those with area-specific projections are called core components and are composed of parvalbumin cells. This classification scheme reveals strong representation of matrix neurons in most ILN subdivisions, but also throughout the thalamus even within primary sensory relay nuclei. Jones $(1998 a, b)$ argues for a diffuse matrix versus core classification of thalamic neurons that limits the selective role of the ILN in engaging multiple cortical regions. This anatomic argument, however, must be weighed along with the unique clinical pathologies associated with the ILN subdivisions, which suggest a preferred role for these paramedian structures in forebrain gating (see also Schiff and Plum [1999]). The main difference demonstrated in this study appears to be in the Cm-Pf complex, possibly indicating its larger role in closed sensorimotor loops (see Smith and Sidibe [1999]).

\section{Physiologic Evidence of Cerebral Gating Processes}

Recent studies have detailed the physiologic connections between the ILN and the MRF, and elucidated further their essential role in both arousal (Glenn and Steriade, 1982; Pare et al., 1988; Steriade and Glenn, 1982) and attentive states (Steriade et al., 1996). Both the $\mathrm{Cm}-\mathrm{Pf}$ (posterior intralaminar group) and the central lateral nucleus may desynchronize the EEG during arousal (Steriade et al., 1990, 1996). Tonic rapid firing in CL correlates with desynchronization of the EEG and responds to inputs from the MRF (Glenn and Steriade, 1982). These studies demonstrate specific changes in the spectral content of background brain activity within different states, including natural awake attentive states, that have been shown to exhibit increased high-frequency activity (Steriade et al., 1996).

It has been proposed, on the basis of magnetoencephalographic recordings and detailed cellular models, that the ILN, in conjunction with the specific thalamic nuclei, establish a thalamocortical resonance at high frequencies (approximately $40 \mathrm{~Hz}$; Llinas and Ribary, 1993; Ribary et al., 1991). Such a global resonance would promote a functional state and would facilitate the distribution and coordination of this activity throughout the forebrain (Llinas and Pare, 1991; Llinas and Ribary, 1993; Llinas et al., 1994; Pare and Llinas, 1995). Phase resets of this system have been proposed to underlie magnetoencephalographic signatures of cognitive binding processes identified during auditory discrimination experiments (Joliot et al., 1994). Steriade et al. $(1993,1996)$ have simultaneously recorded intralaminar thalamic and cortical neurons with synchronous firing in these high-frequency ranges.

Other anatomic and physiologic evidence suggests that the ILN may also play a role in the formation of specific "event-holding" functions that support attention and working memory (Mair, 1994; Purpura and Schiff, 1997; Vogt, 1990; discussed later). Such event-holding functions may represent focal, sustained activations or amplifications of cortical activity (Fuster, 1973), and may be associated with neuronal transient responses (Friston, 1995; Schall, 1991), oscillatory activity (Llinas et al., 1994; Singer and Gray, 1995; Steriade et al., 1996), or possibly other physiologic signatures (Schiff et al., 1999a; Vaadia et al., 1995). Mair (1994) identified 
the possible role of these "event-holding functions," supported by the ILN, in working memory, closely following the original argument by Vogt (1990). Several subsequent experimental results from this research group (Burk and Mair, 1998; Mair et al., 1998; Zhang et al., 1998) have provided evidence that marked deficits in delayed match to sample task performance are attributable to lesions specifically of the ILN (paracentral, central lateral, and central medial, but not the median dorsalis nucleus). These investigators interpreted the observed memory deficits primarily as a disabling of mnemonic functions of CSPTC loops by ILN injury. Through the activation of widely separated local patches of cortex, any particular subdivision of the ILN may facilitate specific long-range cortical-cortical interactions (Amzica et al., 1997; Barth and MacDonald, 1996; Steriade, 1996).

Experimental studies support a selective integrative role for the ILN. Integrative problems in visuospatial awareness are seen with both anterior and posterior thalamic lesions involving the ILN. Circumscribed thalamic lesions thought to be restricted to the $\mathrm{Cm}-\mathrm{Pf}$ complex result in specific impairments in the ability to use extraretinal eye position signals to produce accurate memory-guided saccades (e.g., after a truncal rotation or caloric stimulus, see Gaymard et al. [1994]). Studies of $\mathrm{CL}$ also demonstrate a role in visual awareness. In cats, contraversive head turning and conjugate and contraversive saccadic eye movements are elicited by stimulation of CL (Schlag and Schlag-Rey, 1971). Similarly, unilateral lesions of $\mathrm{CL}$ in cats lead to contralateral visual neglect (Orem et al., 1973).

Schlag-Rey and Schlag (1984a, 1984b) first described a role for the ILN in primate visuospatial awareness. They characterized visuomotor functions in the rostral ILN (primarily CL) of alert monkeys using single-unit recordings in animals performing behavioral tasks. One population of neurons ceased firing during a saccade and then rebounded with a burst of action potentials at the start of the next intersaccadic interval. Most of these neurons demonstrated this behavior for any saccade, with the direction or amplitude of the saccade having no effect on the dynamics of the response. Other neighboring visuomotor units in the ILN (eye position and saccadic burst cells) were highly sensitive to the parameters (amplitude, latency, direction) of the saccade. Schlag-Rey (1984b) and Schlag (1984a) interpreted their findings as militating against the concept of "mass action" in the ILN but hinting at "control signals" or a "clocking device synchronized on saccades used to pace operations at the next stage [of cortical processing]."

Based on the pioneering studies of Schlag-Rey and
Schlag (1984a, 1984b), Purpura and Schiff (1997) proposed that the CL firing responses, sensitive to levels of arousal, have a multifunctional purpose in both setting up large areas of cortical activation and in facilitating more local activations related to visual awareness. ILN neurons are presumed to share intrinsic membrane properties that are common to all thalamic neurons (Jahnsen and Llinas, 1984). Thus, these neurons should be capable of two modes of firing behavior: When hyperpolarized, they should fire a short high-frequency burst superimposed on slower lower amplitude $\mathrm{Ca}++$ spikes. When depolarized, they should fire spikes at a regular rate determined by the level of depolarization. The temporal structure of firing patterns of CL neurons may be controlled both by levels of arousal and visuomotor behavior. Such local activations may be excited by the saccade-related bursts identified by Schlag-Rey (1984b) and Schlag (1984a), thereby facilitating processing in separated cortical regions that receive input during the intersaccadic interval. One requirement of this proposal is that fast bursting neurons must be able to burst in the active depolarized states associated with wakefulness (Steriade, 1997). New evidence from in vivo intracellular recordings during natural sleep-wake cycles shows that such a mechanism may be available (Steriade et al., 1999)

During the tonic firing mode, activation of N-methylD-aspartate channels in the supragranular layer of the cortex may support sustained activity in local populations of cortical pyramidal cells (Larkum et al., 1999; Purpura and Schiff, 1997; Vogt, 1990). In primates engaged in selective attention paradigms, neuronal activity with these characteristics can be recorded from the prefrontal cortex (PFC; Fuster, 1973), frontal eye fields (FEFs; Schall, 1991), and the posterior parietal cortex (PPC; Andersen, 1989; Colby, 1991). In these paradigms, a period of sustained activation in cortical neurons is observed between presentation of a peripheral target and a subsequent saccade to the target's location. Such shifts of attention relate closely to saccadic eye movements. ILN subdivisions selectively project to PFC (Pf, CL, and paralaminar MD), FEF (CL and paralaminar $\mathrm{MD})$, and PPC (Cm-Pf, CL). Through these specific projections they may facilitate and possibly trigger such sustained activations or "event-holding functions" used in visuospatial attention. These sustained neuronal responses may act as "activity envelopes" that gate many different carriers generated by relatively independent local network processes (Schiff et al., 1999a). The transient activation of such activity envelopes may be the basis for episodic cortical processing linking attention and working memory to oculomotor behavior and the intersaccadic interval (Purpura and Schiff, 1997). In the 
PFC, neuronal activations would reflect working memory processing, whereas in the PPC these cortical activations would determine the dynamics of the attentional gate, with the ILN facilitating the welldocumented interdependence of these psychophysical variables (Reeves and Sperling, 1986; Sperling and Weichselgartner, 1995). In FEF these activations would prepare saccadic eye movements to attended targets.

Of note, there is some evidence for similarly selective single-unit behavior in the MRF (Waitzman et al., 1996). Knowledge of the specific functional contributions of the nucleus reticularis in alert humans or primates is very limited outside of interesting attentional and memory failures associated with somewhat selective injury to the NRT after poisoning with domoic acid (Newman, 1997; Teitelbaum et al., 1990) or anoxic injury (Ross and Graham, 1993). Taken together, the main role of the ILN, MRF, and NRT, based on the earlier models of Penfield and Jasper, Skinner and Yingling, and others (as reviewed above), is to facilitate the gating of corticocortical information processing, and not arousal per se (see Fig. 1).

\section{CONCLUSIONS}

As reviewed here, several lines of evidence support a selective role for subcortical gating systems in the mechanism of global disorders, apart from the contributions of the arousal systems. The clinical expression of global disorders produced by subcortical injuries may thus depend on whether such selective gating processes are completely or only partially disabled. For example, the vegetative state represents a complete loss of integrative cerebral activity, as does the brief unconscious state accompanying typical absence seizures. In the absence seizure, the loss of gating may result from several, typically only partially dependent, activity envelopes coalescing into a single "brain beat" (analogous to the bursts of generalized $\Theta$ seen with normal drowsiness). Partial loss of such gating processes would attend hyperkinetic mutism, akinetic mutism, or temporal lobe seizures. In these disorders, preserved but isolated minimal neuropsychological expressions may survive, such as the crude form of attention in akinetic mutism; the uncontrolled, aimless motor intention observed in hyperkinetic mutism; or the automatic behaviors accompa-

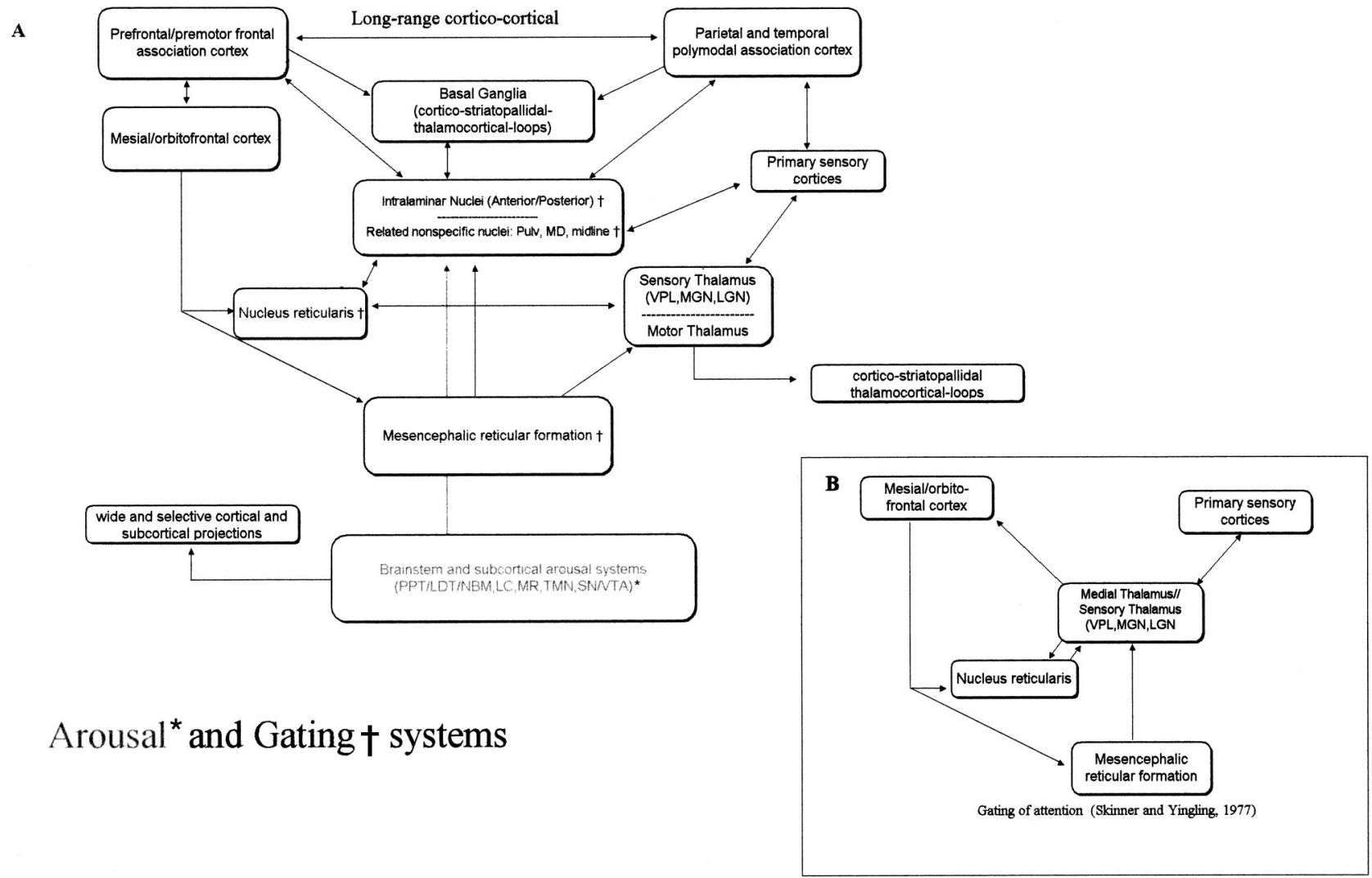

FIG. 1. (A) Schematic overview of brainstem arousal and mesodiencephalic gating systems. (B) Early model of gating of attention by Skinner and Yingling ca. 1977. 
nying complex partial seizures. As suggested earlier, the specific gating processes may be identified with activity envelopes organized around important endogenous transient events, such as eye movements or shifts of attention, that are used to facilitate long-range communication in the forebrain. The episodic dynamics of these activity envelopes, operating within the background arousal state, may be facilitated primarily by the gating systems and may organize many different ongoing neuronal assembly processes.

\section{REFERENCES}

Adams JH, Jennett B, McLellan DR, Murray LS, Graham DI. The neuropathology of the vegetative state after head injury. $J$ Clin Pathol 1999;52:804-6.

Amzica F, Neckelmann, Steriade M. Instrumental conditioning of fast $(20-50 \mathrm{~Hz})$ oscillation in corticothalamic networks. Proc Natl Acad Sci U S A 1997;94:1985-9.

Andersen R. Visual and eye movement functions of the posterior parietal cortex. Anпи Rev Neurosci 1989;12:377-403.

Autret A, Laffont F, de Toffol B, et al. A syndrome of REM and non-REM sleep reduction and lateral gaze paresis after medial tegmental pontine stroke. Computed tomographic scans and anatomical correlations in four patients. Arch Neurol 1988;45:1236 42.

Barth DS, MacDonald KD. Thalamic modulation of high-frequency oscillating potentials in auditory cortex. Nature 1996;383:78-81.

Berendse HW, Groenewegen HJ. Restricted cortical termination fields of the midline and intralaminar thalamic nuclei in the rat. Neuroscience 1991;42:73-102.

Bhatia KP, Marsden CD. The behavioural and motor consequences of focal lesions of the basal ganglia in man. Brain 1994;117:859-76.

Blacklock JB. Effect of barbiturate coma on glucose utilization in normal brain versus gliomas. Positron emission tomography studies. J Neurosurg 1987;67:71-5.

Bogen JE. Some neurophysiologic aspects of consciousness. Semin Neurol 1997;17:95-103.

Brown RG, Marsden CD. Subcortical dementia: the neuropsychological evidence. Neuroscience 1988;25:363-87.

Burk JA, Mair RG. Thalamic amnesia reconsidered: excitotoxic lesions of the intralaminar nuclei, but not the mediodorsal nucleus, disrupt place delayed matching-to-sample performance in rats (Rattus norvegicus). Behav Neurosci 1998:112:54-67.

Cairns H, Olfield RC, Pennybacker JB, et al. Akinetic mutism with an epidermoid cyst of the third ventricle. Brain 1941;64:273-90.

Castaigne $\mathrm{P}$, et al. Demence thalamique d'origine vasculaire par ramollissement bilateral limite au territorie du pedicle retro-mamillaire. Rev Neurol (Paris) 1966;114:89-107.

Castaigne $\mathrm{P}$, et al. Paramedian thalamic and midbrain infarcts: clinical and neuropathological study. Ann Neurol 1981;10;127-48.

Colby CL. The neuroanatomy and neurophysiology of attention. J Child Neurol 1991;6:S90-118.

Contreras D, Destexhe A, Sejnowski TJ, et al. Spatiotemporal patterns of spindle oscillations in cortex and thalamus. J Neurosci 1997; 17:1179-96.

Coull JT, Frith CD, Dolan RJ, et al. The neural correlates of the noradrenergic modulation of human attention, arousal and learning. Eur J Neurosci 1997;9:589-98.

Crick F. Function of the thalamic reticular complex: the searchlight hypothesis. Proc Natl Acad Sci U S A 1984;81:4586-90.

Cummings JL. Subcortical dementia. Br J Psychiatry 1986;149:68297.

Danze F, Brule JF, Haddad K. Chronic vegetative state after severe head injury: clinical study; electrophysiological investigations and CT scan in 15 cases. Neurosurg Rev 1989;12(suppl 1):477-99.

David J, Marathe SB, Patil SD, Grewal RS. Behavioral and electrical correlates of absence seizures induced by thalamic stimulation in juvenile rhesus monkeys with frontal aluminum hydroxide implants: a pharmacologic evaluation. J Pharmacol Methods 1982; 7:219-29.

Delgado-Escueta AV, Bascal FE, Treiman DM. Complex partial seizures on closed circuit television and EEG: a study of 691 attacks in 79 patients. Ann Neurol 1982;11:292-300.

Dennett D. The intentional stance. Cambridge, MA: MIT Press, 1987.

DeVolder AG, et al. Brain glucose metabolism in postanoxic stroke. Arch Neurol 1990;47:197-204.

Dougherty JH Jr, Rawlinson DG, Levy DE, et al. Hypoxic-ischemic brain injury and the vegetative state: clinical and neuropathologic correlation. Neurology 1981;31:991-7.

Dringenberg HC, Vanderwolf $\mathrm{CH}$. Neocortical activation: modulation by multiple pathways acting on central cholinergic and serotonergic systems. Exp Brain 1997;116:160-74.

Facon M, Steriade M, Wertheim N. Hypersomie prolonge engendere par des lesions bilatereles du systeme activateur medial. Le syndrome thrombotique de la bifurcation du tronc basilaire. Rev Neurol (Paris) 1958;98:117-33.

Fisher CM. Honored guest presentation: abulia minor vs. agitated behavior. Clin Neurosurg 1983;31:9-31.

Fleet WS, Valenstein E, Watson RT. Dopamine agonist therapy for neglect in humans. Neurology 1987;37:1765-70.

Fracowiak RSJ, Friston, K, Frith C, Dolan R, Mazziota JC. Human brain mapping. London: Academic Press, 1998.

Friston K. Neuronal transients. Proc R Soc Lond B 1995;261:401-5.

Fromm GH. Role of inhibitory mechanisms in staring spells. J Clin Neurophysiol 1986;3:297-311.

Fuster JM. Unit activity in prefrontal cortex during delayed-response performance: neuronal correlates of transient memory. J Neurophysiol 1973;36:61-78.

Gallup GG. In: Mellgren RL, ed. Animal cognition and behavior. Amsterdam: Noah Holland, 1983:473-510.

Gaymard B, Rivaud S, Pierrot-Deseilligny C. Impairment of extraretinal eye position signals after central thalamic lesions in humans. Exp Brain Res 1994

Glenn LL, Steriade M. Discharge rate and excitability of cortically projecting intralaminar neurons during waking and sleep states. J Neurosci 1982;2:1387-404.

Gloor P, Fariello RG. Generalized epilepsy: some of its cellular mechanisms differ from those of focal epilepsy. Trends Neurosci 1988;11:63-8.

Gokyigit A, Caliskan A. Diffuse spike-wave status of 9-year duration without behavioral change or intellectual decline. Epilepsia 1995; 36:210-3.

Gray CM. The temporal correlation hypothesis of visual feature integration: still alive and well. Neuron 1999;24:31-47.

Groenewegen H, Berendse H. Anatomical relationships between prefrontal cortex and basal ganglia in rat. In: Thierry AM, et al., eds. Motor and cognitive functions of the prefrontal cortex. Berlin: Springer Verlag, 1994a:51-78.

Groenewegen H, Berendse H. The specificity of the 'nonspecific' midline and intralaminar thalamic nuclei. Trends Neurosci $1994 b$ 17:52-66.

Guillery RW, Feig SL, Lozsadi DA. Paying attention to the thalamic reticular nucleus. Trends Neurosci 1981;21:28-32.

Haugeland J. Mind design. Cambridge, MA: MIT Press, 1981.

Helgason C, Wilbur A, Weiss A, Redmond KJ, Kingsbury NA. Acute pseudobulbar mutism due to discrete bilateral capsular infarction in the territory of the anterior choroidal artery. Brain 1988;111: $507-24$.

Huguenard JR. Neuronal circuitry of thalamocortical epilepsy and mechanisms of antiabsence drug action. In: Delgado-Escueta AV, et al., eds. Jasper's basic mechanisms of the epilepsies. 3rd ed., vol. 79. 1999. 
Hunter J, Jasper HH. Effects of thalamic stimulation in unanesthetized animals. Electroencephalogr Clin Neurophysiol 1949;1:305-24.

Inbody S, Jankovic J. Hyperkinetic mutism: bilateral ballism and basal ganglia calcification. Neurology 1986;36:825-7.

Ingvar DH. Reproduction of 3 per second spike and wave EEG pattern by subcortical electrical stimulation in cats. Acta Physiol Scand 1955;33:137-50.

Jahnsen H, Llinas R. Electrophysiological properties of guinea-pig thalamic neurones: an in vitro study. J Physiol 1984;349:205-26.

James W. Principles of psychology. New York: Holt, 1890.

Jasper HH, Droogelever-Fortuyn J. Experimental studies on the functional anatomy of petit mal epilepsy. Res Pub Assist Nerv Ment Disord 1947;26:272-98.

Jasper HH. Current evaluation of concepts of centrencephalic and corticoreticular seizures. Electroencephalogr Clin Neurophysiol 1991;78:2-12.

Jasper H. Consciousness and sensory processing. In: Jasper HH, Descarries L, Castellucci VF, et al., eds. Consciouness at the frontiers of neurology. Vol. 77. Philadelphia: Lippincott Raven, 1998:3348.

Jennett B, Plum F. Persistent vegetative state after brain damage. A syndrome in search of a name. Lancet 1972;1:734-7.

Johnson M. Developmental cognitive neuroscience. Oxford: Blackwell Press, 1997.

Joliot M, Ribary U, Llinas R. Human oscillatory brain activity near 40 $\mathrm{Hz}$ coexists with cognitive temporal binding. Proc Natl Acad Sci U S A 1994;91:11748-51.

Jones E. A new view of the specific and nonspecific thalamocortical connections. In: Jasper HH, Descarries L, Castellucci VF, et al., eds. Consciouness at the frontiers of neurology. Vol. 77. Philadelphia: Lippincott Raven, 1998a.

Jones E. Viewpoint: the core and matrix of thalamic organization. Neuroscience 1998b;85:331-45.

Katz DI, Alexander MP, Mandell AM. Dementia following strokes in the mesencephalon and diencephalon. Arch Neurol 1987; 44:1127-33.

Kinney HC, et al. Neuropathological findings in the brain of Karen Ann Quinlan. N Engl J Med 1994;330:1469-75.

Kinomura S, Larssen J, Gulyas B, et al. Activation by attention of the human reticular formation and thalamic intralaminar nuclei. Science 1996;271:512-5.

Kluver H, Bucy PC. Preliminary analysis of functions of the temporal lobe in monkeys. Arch Neurol Psychiatry 1939;42:979-1000.

Larkum ME, Zhu JJ, Sakmann B. A new cellular mechanism for coupling inputs arriving at different cortical layers. Nature 1999; 398:338-41.

Lavie P, Pratt H, Scharf B, et al. Localized pontine lesion: nearly total absence of REM sleep. Neurology 1984;34:118-20.

Leichnetz GR, Goldberg ME. Higher centers concerned with eye movements and visual attention: corticocortical and thalamic. In: Buttner-Ennever U, ed. Neuroanatomy of the oculomotor system. New York: Elsevier, 1988:365-429.

Levy DE, Sidtis JJ, Rottenberg DA. Differences in cerebral blood flow and glucose utilization in vegetative versus locked-in patients. Ann Neurol 1987;22:673-82.

Llinas R, Pare D. Of dreaming and wakefulness. Neuroscience 1991; 44:521-35.

Llinas R, Ribary U. Coherent $40-\mathrm{Hz}$ oscillation characterizes dream state in humans. Proc Natl Acad Sci U S A 1993;90:2078-81.

Llinas R, et al. Content and context in temporal thalamocortical binding. In: Buzsaki G, et al., eds. Temporal coding in the brain. Berlin: Springer-Verlag, 1994:252-72.

Llinas R, Ribary U, Contreras D, et al. The neuronal basis for consciousness. Philos Trans R Soc Lond B Biol Sci 1998;353:1841-9.

Macchi G. The intralaminar system revisted. In: Miniacchi D, et al., eds. Thalamic networks for relay and modulation. Oxford: Pergamon, 1993:209-28.

Macchi G, Bentivoglio M. The thalamic intralaminar nuclei and the cerebral cortex. In: Jones EG, Peters A, eds. Cerebral cortex. Vol. 5. New York: Plenum Press, 1985:355-89.

Macchi G, Bentivoglio M. Is the 'nonspecific thalamus' still "nonspecific"? Arch Ital Biol 1999;137:201-26.

Mair R. On the role of thalamic pathology in diencephalic amnesia. Rev Neurosci 1994;5:105-40.

Mair RG, Burk JA, Porter MC. Lesions of the frontal cortex, hippocampus, and intralaminar thalamic nuclei have distinct effects on remembering in rats. Behav Neurosci 1998;112:772-91.

Marracco RT, Davidson MC. Neurochemistry of attention. In: Parasuraman R, ed. The attentive brain. Cambridge, MA: MIT Press, 1998:35-50.

Marrocco RT, Witte E, Davidson MC. Arousal systems. Curr Opin Neurobiol 1994;4:166-70.

Martin JJ. Thalamic softening syndromes. In: Steriade M, Jones E, McCormick D. The thalamus. Oxford: Elsevier Publishers, 1997: 653-88.

McCormick DA. Neurotransmitter actions in the thalamus and cerebral cortex and their role in neuromodulation of thalamocortical activity. Prog Neurobiol 1992;39:337-88.

McCormick DA, Bal T. Sleep and arousal: thalamocortical mechanisms. Апnu Rev Neurosci 1997;20:185-215.

Mega MS, Cohenour RC. Akinetic mutism: disconnection of frontalsubcortical circuits. Neuropsychiatry Neuropsychol Behav Neurol 1997; 10:254-9.

Mennemeier M, Crosson B, Williamson DJ, et al. Tapping, talking and the thalamus: possible influence of the intralaminar nuclei on basal ganglia function. Neuropsychologia 1997;35:183-93.

Mikati MA, Holmes GL. Temporal lobe epilepsy. In: Wylie E, ed. The treatment of epilepsy. Philadelphia: Lea and Febiger, 1993:51324.

Mori E, Yamadori A. Rejection behavior: a human homologue of the abnormal behavior of Denny-Brown and Chamber's monkey with bilateral parietal ablation. J Neurol Neurosurg Psychiatry 1989; 52:1260-6.

Morison RS, Dempsey EW. A study of thalamo-cortical relationships. Am J Physiol 1942;135:281-92.

Moruzzi G, Magoun HW. Brainstem reticular formation and activation of the EEG. Electroencephalogr Clin Neurophysiol 1949;1:45573.

Mraovitch S, Calando Y. Limbic and/or generalized convulsive seizures elicited by specific sites in the thalamus. Neuroreport 1995;6:519-25.

Mraovitch S, Calando Y. Interactions between limbic, thalamo-striatal-cortical, and central autonomic pathways during epileptic seizure progression. J Comp Neurol 1999;411:145-61.

Muller A, Baumgartner RW, Rohrenbach C, et al. Persistent KluverBucy syndrome after bilateral thalamic infarction. Neuropsychiatry Neuropsychol Behav Neurol 1999;12:136-9.

Multisociety Task Force on PVS. Medical aspects of the persistent vegetative state. Part 1. N Engl J Med 1994;330:1499-508.

Mesulam M. Behavioral neurology. CNS series. Philadelphia: F.A. Davis, 1983

Nemeth G, Hegedus K, Molnar L. Akinetic mutism associated with bicingular lesions: clinicopathological and functional anatomical correlates. Eur Arch Psychiatry Neurol Sci 1988;237:218-22.

Newman J. Putting the puzzle together. Part II. J Consciousness Studies 1997;4:100-22.

Orem J, Schlag-Rey M, Schlag J. Unilateral visual neglect and thalamic intralaminar lesions int the cat. Exp Neurol 1973;40:78497.

Otto A, Zerr I, Lantsch M, et al. Akinetic mutism as a classification criterion for the diagnosis of Creutzfeldt-Jakob disease. J Neurol Neurosurg Psychiatry 1998;64:524-8.

Panksepp J. Affective neuroscience. Oxford, UK: Oxford University Press, 1998.

Pare D, Llinas R. Conscious and pre-conscious processes as seen from the standpoint of sleep-waking cycle neurophysiology. Neuropsychologia 1995;33:1155-68. 
Pare D, Smith Y, Parent A, et al. Projections of brainstem core cholinergic and non-cholinergic neurons of cat to intralaminar and reticular thalamic nuclei. Neuroscience 1988;25:69-86.

Penfield W. Epileptic automatism and the centrencephalic integrating system. Res Assoc Nerv Ment Disc Proc 1952;30:513-28.

Penfield W. Centrencephalic integrating system. Brain 1958;81:231-4.

Penfield WG, Jasper HH. Epilepsy and the functional anatomy of the human brain. Boston: Little Brown, 1954.

Plum F. Coma and related global disturbances of the human conscious state. In: Jones E, Peters P, eds. Cerebral cortex. Vol. 9. New York: Plenum Press, 1991.

Plum F, Posner J. The diagnosis of stupor and coma. Philadelphia: F. A. Davis, 1966.

Plum F, Posner J. The diagnosis of stupor and coma. 3rd ed. Philadelphia: FA Davis, 1982.

Plum F, Schiff N, Ribary U, et al. Coordinated expression in chronically unconscious persons. Trans $R$ Soc Lond 1998;353:1929-33.

Pollen DA, Perot P, Reid KH. Experimental bilateral wave and spike from thalamic stimulation in relation to level of arousal. Electroencephalogr Clin Neurophysiol 1963;15:1017-28.

Portas CM, Rees G, Howseman AM, et al. A specific role for the thalamus in mediating the interaction of attention and arousal in humans. J Neurosci 1998;18:8979-89.

Posner MI, Rothbart MK. Attention, self regulation and consciousness. Trans $R$ Soc Lond 1998;353:1915-29.

Purpura KP, Schiff ND. The thalamic intralaminar nuclei: role in visual awareness. Neuroscientist 1997;3:8-14.

Reeves A, Sperling G. Attention gating in short-term visual memory. Psychol Rev 1986;93:180-206.

Relkin NR, Petito C, Plum F. Coma and vegetative state secondary to severe anoxic-ischemic damage involving the thalamus. Ann Neurol 1990. Abstract.

Ribary U, Ioannides AA, Singh KD, et al. Magnetic field tomography of coherent thalamocortical $40-\mathrm{Hz}$ oscillations in humans. Proc Natl Acad Sci U S A 1991;88:11037-41.

Ribary U, Schiff N, Kronberg E, et al. Fractured brain function in unconscious humans: functional brain imaging using MEG. Neuroimage 1998;7:S106. Abstract.

Ross DT, Graham DI. Selective loss and selective sparing of neurons in the thalamic reticular nucleus following human cardiac arrest. J Cereb Blood Flow Metab 1993;13:558-67.

Ross ED, Stewart RM. Akinetic mutism from hypothalamic damage: successful treatment with dopamine agonists. Neurology 1981;31: $1435-9$.

Rudolf J, Ghaemi M, Ghaemi M, et al. Cerebral glucose metabolism in acute and persistent vegetative state. J Neurosurg Anesthesiol 1999;11:17-24

Sarter M, Bruno JP. Cortical cholinergic inputs mediating arousal, attentional processing and dreaming: differential afferent regulation of the basal forebrain by telencephalic and brainstem afferents. Neuroscience 2000;95:933-52.

Scannell JW, Burns GA, Hilgetag CC, et al. The connectional organization of the cortico-thalamic system of the cat. Cereb Cortex 1999;9:277-99.

Schall JD. Neuronal activity related to visually guided saccades in the frontal eye fields of rhesus monkeys: comparison with supplementary eye fields. J Neurophysiol 1991;66:559-79.

Schiebel AB. Anatomical and physiological substrates of arousal: view from the bridge. In: Hobson JA, Brazier MAB, eds. The reticular formation revisted. New York: Raven Press, 1980:55-66.

Schiebel ME, Schiebel AB. The organization of the nucleus retiuclaris: a golgi study. Brain Res 1966;1:43-62.

Schiff ND, Labar DL, Victor JD. Common dynamics in temporal lobe and absence seizures. Neuroscience 1999a;91:417-28.

Schiff ND, Plum F. Target article. The neurology of impaired consciousness: global disorders and implied models. Association for Scientific Study of Consciousness Electronic Seminar Series. 1999. http://athena.english.vt.edu/cgi-bin/netforum/nic/a/l.

Schiff ND, Purpura KP, Victor JD. Gating of local network signals appear as stimulus-dependent activity envelopes in striate cortex. J Neurophysiol 1999b;82:2182-96.

Schiff ND, Ribary U, Plum F, et al. Words without mind. $J$ Cogn Neurosci $1999 c ; 11: 650-6$.

Schlag J, Schlag-Rey M. Induction of oculomotor responses from thalamic internal medullary lamina in the cat. Exp Neurol 1971; 33:498-508.

Schlag-Rey M, Schlag J. Visuomotor functions of central thalamus in monkey. I. Unit activity related to spontaneous eye movements. J Neurophysiol 1984a;40:1149-74.

Schlag-Rey M, Schlag J. Visuomotor functions of central thalamus in monkey. II. Unit activity related to visual events, targeting and fixation. J Neurophysiol 1984b;40:1175-95.

Searle J. Intentionality: an essay in philosophy of mind. Cambridge, UK: Cambridge University Press, 1983.

Segarra JM. Cerebral vascular disease and behavior. The syndrome of the mesencephalic artery (basilar artery bifurcation). Arch Neurol 1970;22:408-18.

Silberstein R. Neuromodulation of neocortical dynamics. In: Nunez P, ed. Neocortical dynamics and human EEG rhythms. Oxford, UK: Oxford University Press, 1995:521-622.

Singer W. Neuronal synchrony: a versatile code for the definition of relations? Neuron 1999;24:49-65.

Singer W, Gray C. Visual feature integration and the temporal correlation hypothesis. Апnи Rev Physiol 1995;55:349-74.

Skinner JE, Yingling CD. Central gating mechanisms that regulate event-related potentials and behavior. In: Desmedt JE, ed. Progress in clinical neurophysiology. Vol. 1. Attention, voluntary contraction, and event-related cerebral potentials. Basel: Karger, 1977:70-96.

Smiley JF, Subramanian M, Mesulam MM. Monaminergic-cholinergic interactions in the primate basal forebrain. Neuroscience 1999:93:817-29.

Smith Y, Sidibe M. Functional interconnections between the caudal intralaminar nuclei and the striatopallidal complex in monkeys. Presented at the 29th annual meeting of the Society for Neuroscience. Miami, FL. Abstract no. 764.20. 1999.

Snead OC. Basic mechanisms of generalized absence seizures. Ann Neurol 1995;37:146-57.

Sperling G, Weichselgartner E. Episodic theory of the dynamics of spatial attention. Psychol Rev 1995;102:503-32.

Spiegel EA, Wycis HT, Orchink C, et al. Thalamic chronotaraxis. Am J Psychiatry 1956;113:97-105.

Steriade M. Interneuronal epileptic discharges related to spike-andwave conical seizure in behaving monkeys. Electroencephalogr Clin Neurophysiol 1974;37:247-63.

Steriade M. Arousal: revisiting the reticular activating system. Science 1996;272:225-6.

Steriade M. Thalamic substrates of disturbances in states of vigilance and consciousness in humans. In: Steriade M, Jones E, McCormick D, eds. Thalamus. Oxford, UK: Elsevier Publishers, 1997: $721-42 .$.

Steriade M. Invited commentary. Disorders of consciousness. Association for Scientific Study of Consciousness Electronic Seminar Series. 1999. http://athena.english.vt.edu/cgi-bin/netforum/nic/a/ 14-1.2.2.

Steriade M, Amzica F, Contreras D. Synchronization of fast 30-40 Hz spontaneous cortical rhythms during brain activation. $J$ Neurosci 1996;16:392-417.

Steriade M, Buzsaki, G. Parallel activation of thalamic and cortical neurons by brainstem and basal forebrain cholinergic systems. In: Steriade M, Biesold D, eds. Brain cholinergic system. New York: Oxford University Press, 1990.

Steriade M, Contreras D. Relations between cortical and thalamic cellular events during transition from sleep patterns to paroxysmal activity. J Neurosci 1995;15:623-42.

Steriade M, Curro Dossi R, Contreras D. Electrophysiological properties of intralaminar thalamocortical cells discharging rhythmic (approximately $40 \mathrm{~Hz}$ ) spike-bursts at approximately $1000 \mathrm{~Hz}$ 
during waking and rapid eye movement sleep. Neuroscience 1993;56:1-9.

Steriade M, Glenn LL. Neocortical and caudate projections of intralaminar thalamic neurons and their synaptic excitation from midbrain reticular core. J Neurophysiol 1982;48:352-71.

Steriade M, Jones E, McCormick D, eds. Thalamus. Oxford UK: Elsevier Publishers, 1997.

Steriade M, Llinas RR. The functional states of the thalamus and the associated neuronal interplay. Physiol Rev 1988;68:649-742.

Steriade M, Timofeev I, Grenier F. Intracellular activity of various neocortical cell-clasess during the natural sleep-wake cycle. Presented at the 29th annual meeting of the Society for Neuroscience. Miami, FL. Abstract no. 664.14. 1999.

Stuss DT, Guberman A, Nelson R, et al. The neuropsychology of paramedian thalamic infarction. Brain Cogn 1988;8:348-78.

Sukov W, Barth DS. Three-dimensional analysis of spontaneous and thalamically evoked gamma oscillations in the auditory cortex. J Neurophysiol 1998;79:2875-84.

Teitelbaum JS, et al. Neurologic sequelae of domoic acid intoxication due to the ingestion of contaminated mussels. $N$ Engl $\mathrm{J}$ Med 1990;322:1781-7.

Thompson R. Centrencephalic theory, generalized learning system and subcortical dementia. In: Crinella F, Yu, J, eds. Brain mechanisms. New York: New York Academy of Sciences, 1993:197-224.

Tomassino C, Grana C, Lucignani G, et al. Regional metabolism of comatose and vegetative state patients. J Neurosurg Anesthesiol 1995;7:109-16.

Vaadia E, et al. Dynamics of neuronal interactions in monkey cortex in relation to behavioural events. Nature 1995;373:515-8.

Van Der Werf YD, Mitter, MP. Are the midline nuclei of the thalamus involved in cognitive functioning? A neuroanatomical account. Society for Neuroscience 29th Annual Meeting, Miami, FL. Abstract, 751.2, 1999. van Domburg PH, ten Donkelaar HJ, Notermans SL. Akinetic mutism with bithalamic infarction. Neurophysiological correlates. $\mathrm{J} \mathrm{Neu}$ rol Sci 1996;139:58-65.

Velasco F, Velasco M, Marquez I, et al. Role of centromedian thalamic nucleus in the genesis, propagation, and arrest of epileptic activity. An electrophysiological study in man. Acta Neurochir 1993; 58(suppl):201-4.

Vogt BA. The role of layer 1 in cortical function. In: Jones EG, Peters A, eds. Cerebral cortex. Vol. 9. New York: Plenum Press, 1990:49-80.

Von der Malsburg C. The what and why of binding: the modeler's perspective. Neuron 1999;24:95-104.

Waitzman DM, Silakov VL, Cohen B. Central mesencephalic reticular formation (cMRF) neurons discharging before and during eye movements. J Neurophysiol 1996;75:1546-72.

Walshe FMR. The brainstem conceived as the 'highest level' of function in the nervous system: with particular reference to the 'automatic apparatus' of Carpenter (1850) and to the 'centrencephalic integrating system' of Penfield. Brain 1957;80:510-39.

Watt D. Emotions and consciousness: implications of affective neuroscience for extended reticular thalamic activating system theories of consciousness. ASSCE seminar target article. 1998. http:// server.phil.vt.edu/assc/watt/default.html.

Weese GD, Phillips JM, Brown VJ. Attentional orienting is impaired by unilateral lesions of the thalamic reticular nucleus in the rat. J Neurosci 1999;19:10135-9.

Yeterian EH, Pandya DN. Thalamic connections of the superior temporal sulcus in the rhesus monkey. J Comp Neurol 1989;282: $80-97$.

Zhang Y, Burk JA, Glode BM, Mair RG. Effects of thalamic and olfactory cortical lesions on continuous olfactory delayed nonmatching-to-sample and olfactory discrimination in rats (Rattus norvegicus). Behav Neurosci 112:39-53. 